\title{
Shadow-Mask Evaporation through Monolayer-Modified Nanostencils
}

\author{
Marius Kölbel, † R. Willem Tjerkstra, ${ }^{\ddagger}$ Jurgen Brugger, ${ }^{\S}$ Cees J. M. van Rijn," \\ Wietze Nijdam," Jurriaan Huskens, ${ }^{*, \dagger}$ and David N. Reinhoudt ${ }^{*}, \dagger$ \\ Laboratory of Supramolecular Chemistry and Technology and Transduction \\ Technology Group, MESA + Research Institute, University of Twente, \\ P.O. Box 217, 7500 AE Enschede, The Netherlands, Microsystems Laboratory, \\ École Polytechnique Fédérale de Lausanne, 1015 Lausanne, Switzerland, and \\ Aquamarijn Micro Filtration B.V., Beatrixlaan 2, 7255 DB Hengelo Gld., \\ The Netherlands
}

Received September 6, 2002

\begin{abstract}
Gradual clogging of the apertures of nanostencils used as miniature shadow masks in metal evaporations can be reduced by coating the stencil with self-assembled monolayers (SAM). This is quantified by the dimensions (height and volume) of gold features obtained by nanostencil evaporation as measured by scanning electron microscopy (SEM) and atomic force microscopy (AFM). An increase in material deposition through the apertures by more than $100 \%$ can be achieved with SAM-coated stencils, which increases their lifetime.
\end{abstract}

The nanostencil technique (Figure 1) is a promising new tool for the generation of submicrometer and nanopatterns. It makes use of a thin silicon nitride membrane with circular, rectangular, or line-shaped apertures fabricated using optical lithography, E-beam lithography (EBL), or the focused ion beam (FIB) technique. ${ }^{1}$ This membrane serves as a shadow mask in the HV or UHV physical vapor deposition (PVD) of various materials such as metals, semiconductors, dielectrics, and organics. Complex patterns can thus be realized in a resistless, single-step process. The combination of stencil mask evaporation with scanning probe methods allows the movement of the substrate relative to the stencil and thus the generation of various patterns with a single mask. ${ }^{2}$ Rapid patterning of various surfaces for applications in nanoelectronics, molecular electronics, or bioanalytics is significantly facilitated by this technique.

Shadow-mask evaporation is accompanied by gradual aperture clogging (Figure 1). It has been reported that for sub-100-nm openings a feature thickness of about 3 times the aperture diameter can be achieved before the hole is completely clogged by gold. ${ }^{3}$ At this point, the high-vacuum process has to be interrupted to clean or replace the stencila time-consuming break in the working procedure, which

\footnotetext{
* Corresponding authors. E-mail: j.huskens@ct.utwente.nl; d.n.reinhoudt@ct.utwente.nl.

$\dagger$ Laboratory of Supramolecular Chemistry and Technology, MESA+ Research Institute, University of Twente.

$\doteqdot$ Transduction Technology, MESA + Research Institute, University of Twente.

§ École Polytechnique Fédérale de Lausanne.

"Aquamarijn Micro Filtration B.V.
}

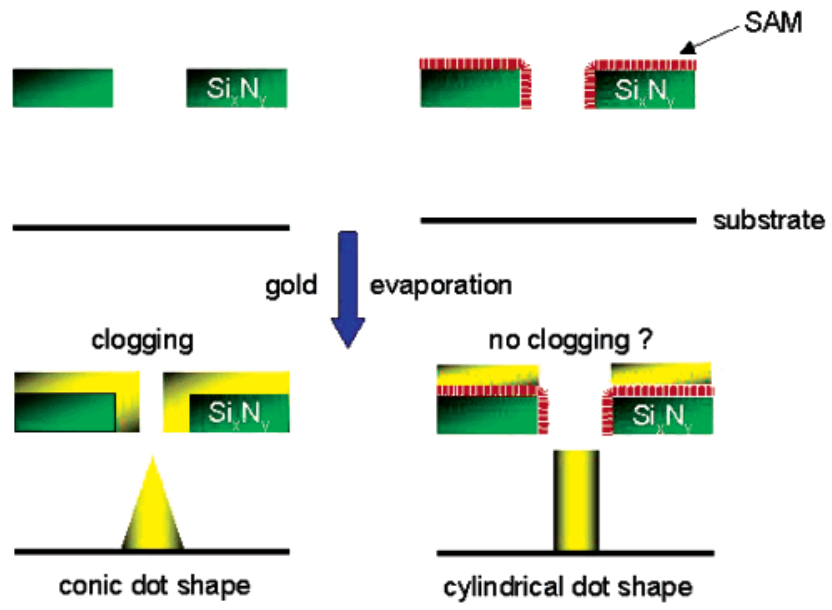

Figure 1. Principle of the nanostencil technique.

should be reduced as much as possible. Suppressing the adhesion of evaporated material to the walls of the stencil apertures is a way to face this problem.

In recent studies, we investigated the influence of alkyl and perfluoroalkyl self-assembled monolayer (SAM) coatings on the adhesion properties of nanostencils. ${ }^{4,5} \mathrm{We}$ could show, on the basis of SEM images, that coating nanostencils with SAMs remarkably reduces the adhesion of gold inside the apertures. The SAMs are not damaged by bombardment with high-energy atoms in HV under various angles, as was established by contact-angle goniometry. They also proved to be stable against $\mathrm{I}_{2} / \mathrm{KI}$ gold-etching solutions, a fact that 


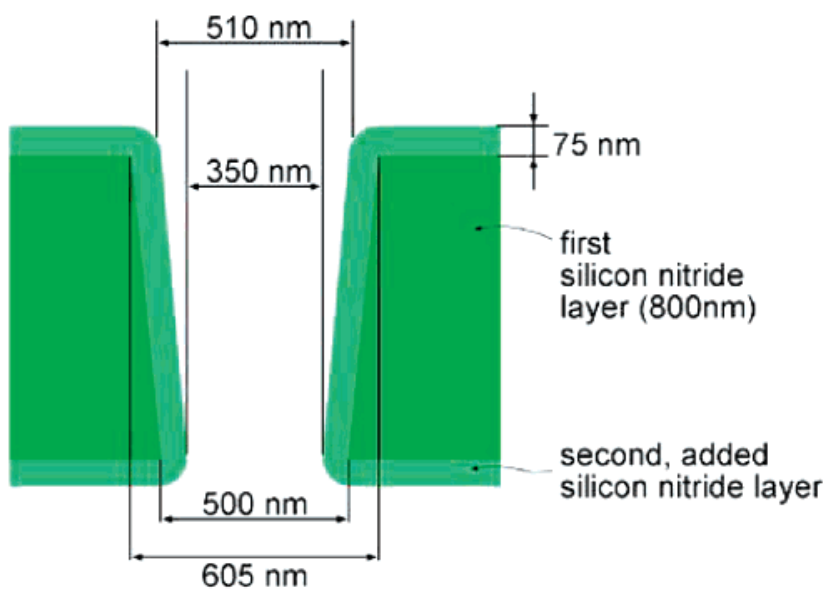

Figure 2. Cross section of a nanostencil aperture after reduction of the diameter from 500 to $350 \mathrm{~nm}$ by LPCVD.

Scheme 1

\begin{tabular}{|c|c|}
\hline CF-Si(OEt) $)_{3}$ & $\stackrel{\text { OEt }}{\text { EtO-Si }} \underset{\text { OEt }}{\text { Si }} \mathrm{C}_{8} F_{1}$ \\
\hline $\mathrm{C} 12-\mathrm{SiCl}_{3}$ & 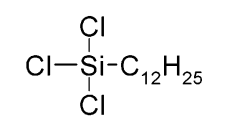 \\
\hline C12-Si(OEt) ${ }_{3}$ & $\begin{array}{c}\text { OEt } \\
\text { EtO- } \\
\text { Si- } \mathrm{C}_{12} \mathrm{H}_{25} \\
\text { OEt }\end{array}$ \\
\hline
\end{tabular}

allows the gold to be easily removed repeatedly by wet etching, leaving the coating intact.

In this contribution, we will give a quantitative analysis of the coating effect on the dimensions of the evaporated patterns and elucidate the details of the clogging mechanism.

The fabrication process for the nanostencils with $500-\mathrm{nm}$ apertures can be found in ref 5 . These stencils have been used to fabricate the stencils with $350-\mathrm{nm}$ apertures by the postprocess growth of additional layers as follows. A wafer containing an array of 500-nm nanostencils was cleaned (5 min in $100 \% \mathrm{HNO}_{3}$ followed by $15 \mathrm{~min}$ in $70 \% \mathrm{HNO}_{3}$ at $\left.70{ }^{\circ} \mathrm{C}\right)$. Subsequently, a thin layer $(75 \mathrm{~nm})$ of silicon nitride was applied by means of low-pressure chemical vapor deposition $\left(\mathrm{SiH}_{2} \mathrm{Cl}_{2} / \mathrm{NH}_{3}=70: 18\right.$ at $\left.850{ }^{\circ} \mathrm{C}, 250 \mathrm{mbar}\right)$. This process yields a very good step coverage such that the initial pore size of $500 \mathrm{~nm}$ is reduced to $350 \mathrm{~nm}$ (see Figure 2 for the cross section of a single pore). Finally, the wafer was diced at a rate of $2 \mathrm{~mm} / \mathrm{s}$ (to avoid damage) into $6 \mathrm{~mm} \times 6$ $\mathrm{mm}$ square pieces, which were used as the nanostencils in the experiments. All silicon particles that were released during dicing were removed by cleaning the samples in a $25 \% \mathrm{KOH}\left(70{ }^{\circ} \mathrm{C}\right)$ and a $5 \% \mathrm{H}_{2} \mathrm{SO}_{4}$ solution $\left(40{ }^{\circ} \mathrm{C}\right)$. The holes in the membrane thus obtained have tapered cross sections. Whereas on one side their diameter corresponds to the nominal values, they amount to $510 \pm 20 \mathrm{~nm}$ and 605 $\pm 35 \mathrm{~nm}$, respectively, on the other side.

The coating of the nanostencils with SAMs of compounds $\mathrm{CF}-\mathrm{Si}(\mathrm{OEt})_{3}, \mathrm{C} 12-\mathrm{SiCl}_{3}$, and $\mathrm{C} 12-\mathrm{Si}(\mathrm{OEt})_{3}$ (Scheme 1) was performed as described earlier. ${ }^{5}$

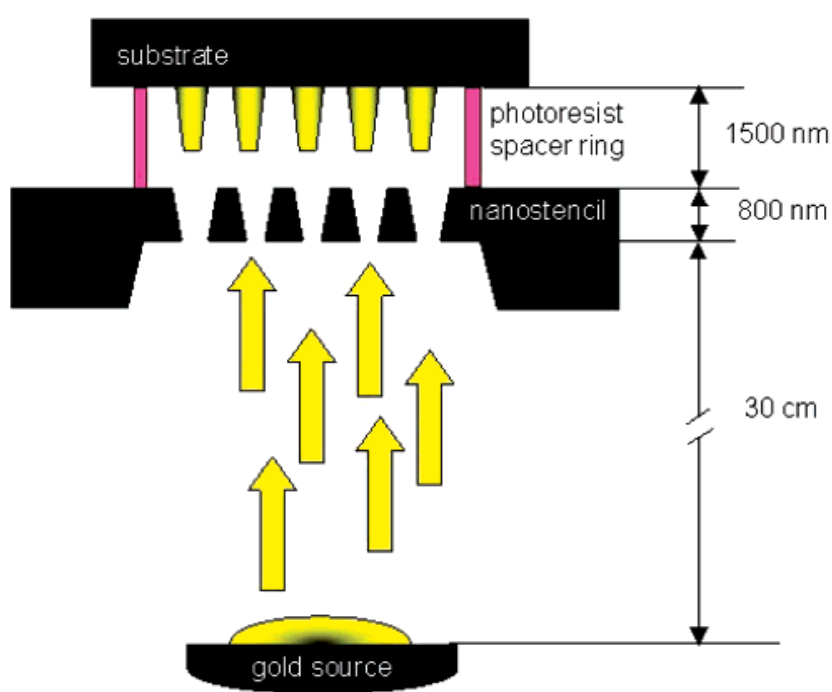

Figure 3. Geometrical paramters of the evaporation experiments.

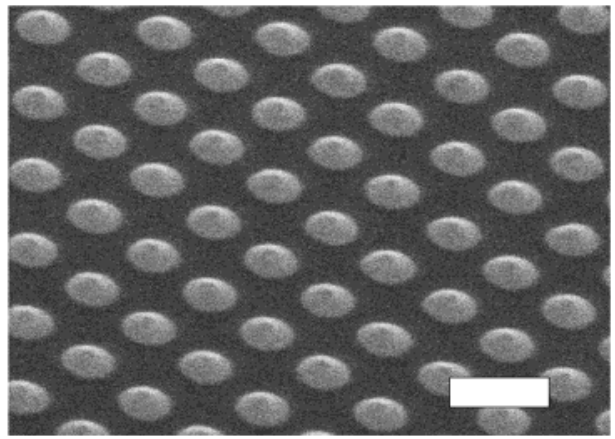

Figure 4. Au dots (150 nm thick) obtained by evaporation through a $\mathrm{C} 12-\mathrm{SiCl}_{3}$-coated stencil in experiment $\mathrm{A}(15.3 \mathrm{Kx}$, scale bar: $1.3 \mu \mathrm{m})$.

Table 1: Stencil Aperture Diameters and Properties of Gold Dots Obtained from SEM and AFM Pictures

\begin{tabular}{llrrrrrr}
\hline expt & \multicolumn{1}{c}{ coatings } & $\begin{array}{c}\mathrm{d}_{\mathrm{a}} \\
(\mathrm{nm})^{\mathrm{a}}\end{array}$ & $\begin{array}{c}\mathrm{d} \\
(\mathrm{nm})^{\mathrm{b}}\end{array}$ & $\begin{array}{c}\mathrm{h} \\
(\mathrm{nm})^{\mathrm{c}}\end{array}$ & $\begin{array}{c}\mathrm{V}_{\text {the }} \\
\left(10^{6} \mathrm{~nm}^{3}\right)^{\mathrm{d}}\end{array}$ & $\begin{array}{c}\mathrm{V}_{\text {exp }} \\
\left(10^{6} \mathrm{~nm}^{3}\right)^{\mathrm{e}}\end{array}$ & $\begin{array}{c}\mathrm{V}_{\text {exp }} / \\
\mathrm{V}_{\text {the }}\end{array}$ \\
\hline A & uncoated & 497 & 708 & 65 & 29.1 & 8.5 & 0.29 \\
& CF & 530 & 634 & 147 & 33.1 & 15.5 & 0.47 \\
& $\mathrm{C} 12-\mathrm{SiCl}_{3}$ & 507 & 764 & 138 & 30.3 & 21.1 & 0.70 \\
$\mathrm{~B}$ & uncoated & 570 & 636 & 825 & 255 & 87 & 0.34 \\
& CF-Si(OEt $)_{3}$ & 590 & 684 & 897 & 273 & 110 & 0.40 \\
C & uncoated & 640 & 642 & 1016 & 322 & 110 & 0.34 \\
& C12-Si(EtO) & 620 & 667 & 950 & 302 & 111 & 0.37
\end{tabular}

${ }^{a} d_{\mathrm{a}}$ : aperture diameter (SEM). ${ }^{b} d$ : dot diameter (SEM). ${ }^{c} h$ : dot height (AFM). ${ }^{d} V_{\text {the }}$ : theoretical dot volume (cylinder). ${ }^{e} V_{\text {exp }}$ : experimental dot volume (cone)

The two or three stencils used together in one experiment belonged to the same batch, which means that the average aperture diameter, aperture shape, and membrane thickness are identical. Silicon squares with dimensions of $1 \mathrm{~cm} \times 1$ $\mathrm{cm}$ were used as the substrates. A $1.5-\mu \mathrm{m}$-thick photoresist spacer ring was prepared on the substrates to maintain a constant distance between the substrate and the mask in all experiments, to prevent the $1-\mu \mathrm{m}$-high dots from growing into the stencil mask, and to provide a well-defined gap between the stencil and the substrate. The nanostencils were 
a

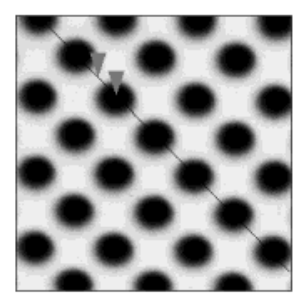

b

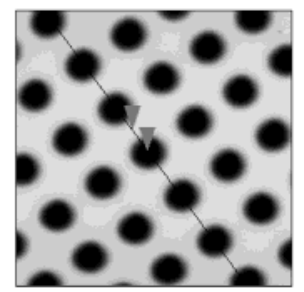

C

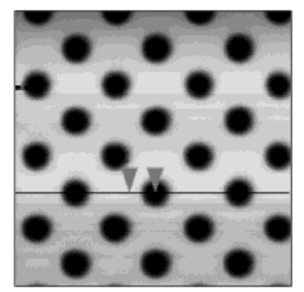

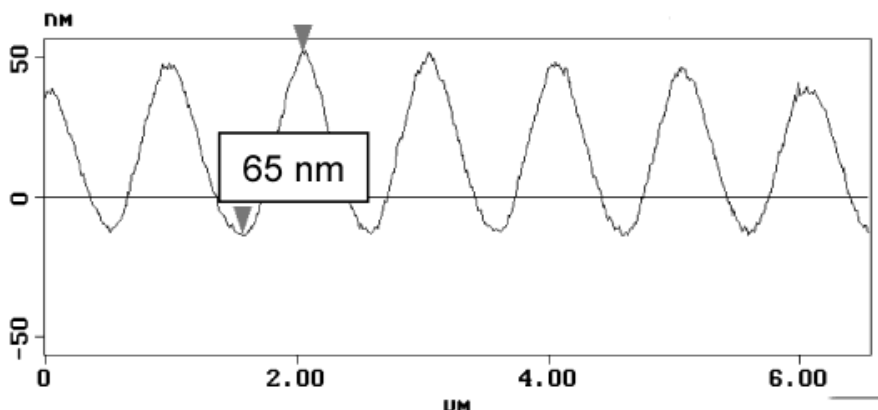
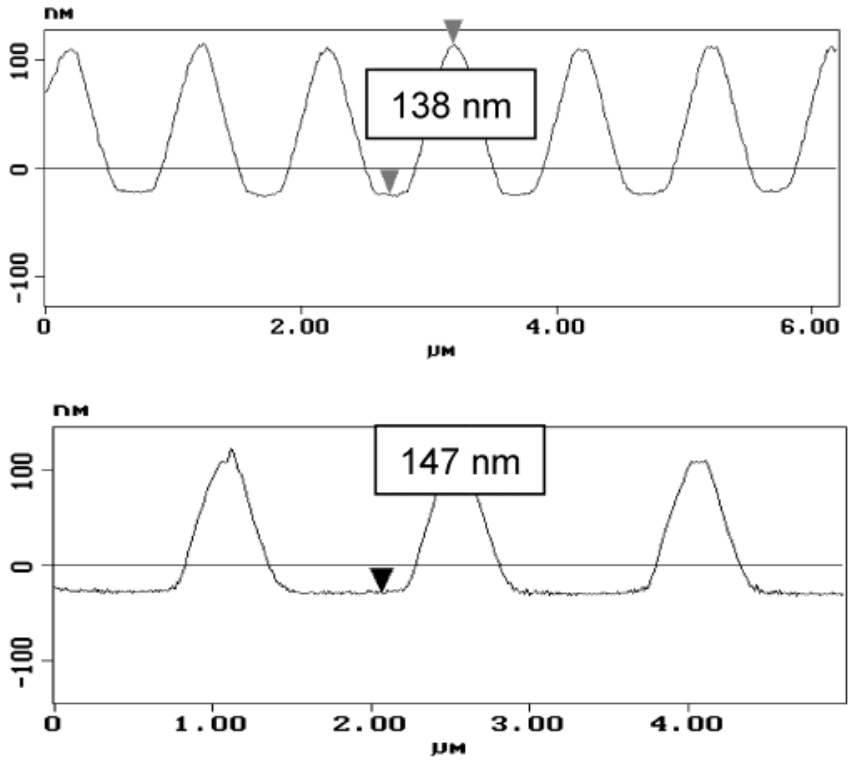

Figure 5. AFM images $(5 \mu \mathrm{m} \times 5 \mu \mathrm{m})$ and section analysis for the gold dots obtained in experiment A using an (a) uncoated, (b) $\mathrm{C} 12-\mathrm{SiCl}_{3}$-coated, and (c) $\mathrm{CF}-\mathrm{Si}(\mathrm{OEt})_{3}$-coated nanostencil.

clamped on top of the substrates in a special sample holder. ${ }^{5}$ The face of the stencil having the larger aperture diameter was positioned toward the substrate, with the opposite face positioned toward the gold source (Figure 3). The sample holder was placed in a Balzers BA 510 thermal evaporator $30 \mathrm{~cm}$ above and at an angle of $\sim 5^{\circ}$ to the evaporation source. The chamber was evacuated to $\sim 5 \times 10^{-6} \mathrm{mbar}$ before the evaporation was started. The pressure during evaporation was $\sim 5 \times 10^{-5}$ mbar, the voltage was $90 \mathrm{~V}$, and the evaporation rate was $\sim 24 \mathrm{~nm} / \mathrm{min}$. The change in the thickness of the gold layer that formed was monitored by means of an internal quartz crystal microbalance (QCM).

We first carried out an experiment (A) with the evaporation of $150 \mathrm{~nm}$ of gold through an uncoated, a $\mathrm{CF}-\mathrm{Si}(\mathrm{OEt})_{3^{-}}$ coated, and a $\mathrm{C} 12-\mathrm{SiCl}_{3}$-coated nanostencil with $350-\mathrm{nm}$ apertures. After the evaporations, the resulting gold dot patterns on the substrates were investigated by SEM and tapping-mode AFM. SEM images were taken with a Hitachi S800 FEG-SEM. Tapping-mode AFM measurements were performed with a Digital Instruments multimode AFM equipped with a Nanoscope 3 controller.

Figure 4 shows an SEM image of 150-nm-thick gold dots obtained by evaporation through a $\mathrm{C} 12-\mathrm{SiCl}_{3}$-coated nanostencil with $350-\mathrm{nm}$ apertures. The same feature shape is observed for the dots obtained using uncoated or $\mathrm{CF}-$ $\mathrm{Si}(\mathrm{OEt})_{3}$-coated stencils in experiment $\mathrm{A}$.

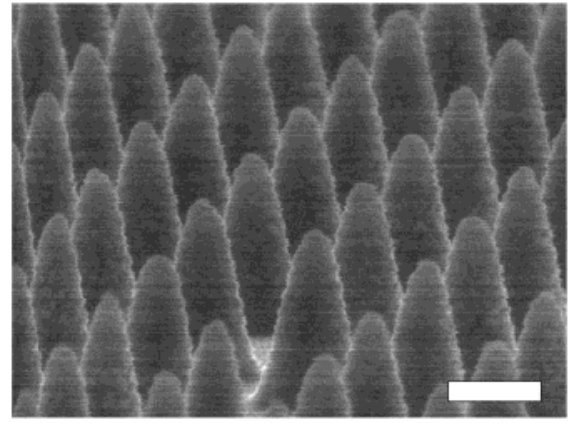

Figure 6. Au dots (1 $\mu \mathrm{m}$ high) obtained by evaporation through a $\mathrm{CF}-\mathrm{Si}(\mathrm{OEt})_{3}$-coated stencil in experiment $\mathrm{B}(40 \mathrm{Kx}$, scale bar: $500 \mathrm{~nm})$.

In Table 1, the dimensions of the dots obtained in experiment $\mathrm{A}$ are summarized. Immediately, a striking difference becomes apparent: the dots obtained using a $\mathrm{CF}-$ $\mathrm{Si}(\mathrm{OEt})_{3}$ or $\mathrm{C} 12-\mathrm{SiCl}_{3}$-coated stencil are more than 2 times higher than the ones obtained with an uncoated stencil, according to AFM measurements (Figure 5). This height difference is also confirmed by measurements on fractured samples studied by SEM under an angle of $90^{\circ}{ }^{6}{ }^{6} \mathrm{We}$ also calculated the volume of the dots assuming a perfect cone shape and using the diameter as measured by $\mathrm{SEM}^{7}$ and the height as measured by AFM. ${ }^{8}$ Clearly, the volume of the features obtained using SAM-coated stencils is much larger 

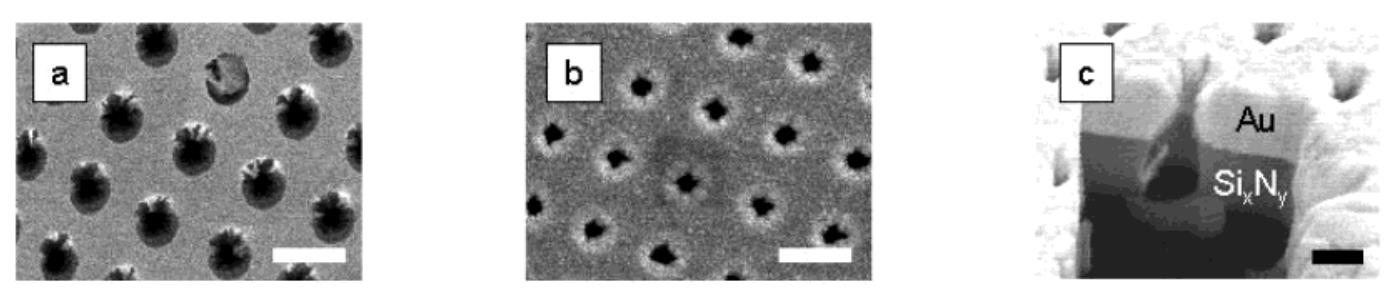

Figure 7. $\mathrm{SEM}$ images of a $\mathrm{CF}-\mathrm{Si}(\mathrm{OEt})_{3}$-coated stencil after the evaporation of $1-\mu \mathrm{m} \mathrm{Au}$ : (a) face positioned toward the substrate $(25$ $\mathrm{Kx}$, scale bar: $1 \mu \mathrm{m})$, (b) face positioned toward the Au source $(25 \mathrm{Kx}$, scale bar: $1 \mu \mathrm{m}$ ), and (c) FIB cross section through the stencil (35 $\mathrm{Kx}$, scale bar: $500 \mathrm{~nm}$ ).

than that of the dots generated with the uncoated mask. Table 1 also gives the expected dot volume $V_{\text {the }}$ (calculated for an ideal cylinder on the basis of the aperture diameter as measured by SEM and the nominal height as measured by the internal QCM) that should be obtained when neither diffusion nor clogging nor scattering takes place. In the last column, the ratio of the experimental to theoretical volume is specified.

These results confirm the remarkable ability of SAM coatings to reduce the adhesion of evaporated material inside the apertures of the stencils. Whereas more than $70 \%$ of the evaporated gold sticks to the aperture walls of an uncoated silicon nitride stencil, only $30 \%$ of the material adheres to those of a $\mathrm{C} 12-\mathrm{SiCl}_{3}$-coated shadow mask.

In a second experiment (B), we evaporated $1 \mu \mathrm{m}$ of gold through an uncoated and a $\mathrm{CF}-\mathrm{Si}(\mathrm{OEt})_{3}$-coated stencil with 500-nm apertures, and in a third experiment (C), we evaporated $1 \mu \mathrm{m}$ of gold through an uncoated and a C12$(\mathrm{OEt})_{3}$-coated $^{9}$ stencil with 500 -nm apertures.

The conic shape of the gold dots in Figure 6, obtained by evaporation through a $\mathrm{CF}-\mathrm{Si}(\mathrm{OEt})_{3}$-coated stencil, is representative of the $1-\mu \mathrm{m}$ features obtained by evaporation through a stencil mask with $500-\mathrm{nm}$ apertures. ${ }^{10}$

Table 1 summarizes the geometrical parameters of experiments $\mathrm{B}$ and $\mathrm{C}$ and the dimensions of the features obtained. The stencils employed in experiment B are not identical to those used in experiment $\mathrm{C}$. The differences in feature height and in the $d_{\mathrm{a}} / d$ ratio between the two experiments can therefore be ascribed to slight variations in membrane thickness, aperture shape, and experimental conditions (pressure, evaporation energy).

Interestingly, the dimensions of the $1-\mu \mathrm{m}$ dots differ from each other much less than those of the 150-nm dots. Although the features generated using SAM-coated nanostencils still have somewhat higher volumes compared to those made with uncoated stencils, the effect is only marginal.

What is the cause of these observations? During the evaporation, a thick gold layer is formed on the face of the nanostencil oriented toward the gold source. The diameters of the openings on the surface of this gold layer are much smaller than those of the apertures on the stencil face that had been oriented toward the substrate. The comparison is shown in Figure 7a and $b$. The effect is also clearly confirmed by SEM images such as that in Figure 7c, in which the conic shape of the pores in the gold layer has been made visible by cutting a cross section using FIB milling (FIB 200, FEI Company). The gradual clogging, giving rise to the cone shape of the gold dots, obviously occurs in the gold layer rather than inside the stencil apertures.

A comparison of the results of experiment A with those of experiments $\mathrm{B}$ and $\mathrm{C}$ convincingly demonstrate the capacity and limitations of shadow-mask evaporations using SAM-coated nanostencils. For low amounts of evaporated material (on the order of 150-nm thickness), the deposition of gold onto the walls of the apertures (which can be reduced by SAM coatings) strongly affects the rate of dot formation. For higher amounts, with increasing thickness of the gold layer on top of the stencil, the narrowing of the aperture within the gold layer becomes the determining factor. Thus, at first glance, the effect of the coating seems rather limited. However, for smaller apertures $(<100 \mathrm{~nm})$ and small amounts of evaporated material (feature heights of $10 \mathrm{~nm}$ ), as envisaged for future nanotechnological applications, it is very likely to improve the lifetime of the mask. Increasing the ratio between evaporation thickness and aperture diameter by a factor of 2 or 3 represents a significant enhancement of throughput of the nanostencil technique.

We demonstrated that SAM coatings reduce the adhesion of gold inside the apertures of silicon nitride nanostencil membranes significantly. A comparison of the dot dimensions (height and volume) obtained with coated and uncoated masks confirms this expected effect. For an evaporation thickness of $150 \mathrm{~nm}$, an increase in throughput to as much as $240 \%$ can be achieved with coated stencils. At much higher amounts of evaporated material, clogging of the holes in the gold layer building up on top of the mask, rather than inside the apertures of the stencil, becomes the limiting factor. Nevertheless, for the generation of sub-100-nm features, SAM coating of the nanostencils has high potential.

Acknowledgment. We thank Mark A. Smithers for SEM images and Frans Segerink for FIB experiments. This work was supported by the EU in the 5FP IST project ATOMS.

\section{References}

(1) Brugger, J.; Berenschot, J. W.; Kuiper, S.; Nijdam, W.; Otter, B.; Elwenspoek, M. Microelectron. Eng. 2000, 53, 403-405. Schlittler, R. R.; Seo, J. W.; Gimzewski, J. K.; Durkan, C.; Saifullah, M. S. M.; Welland, M. E. Science (Washington, D.C.) 2001, 292, 11361139.

(2) Lüthi, R.; Schlittler, R. R.; Brugger, J.; Vettiger, P.; Welland, M. E.; Gimzewski, J. K. Appl. Phys. Lett. 1999, 75, 1314-1316.

(3) Deshmukh, M. M.; Ralph, D. C.; Thomas, M.; Silcox, J. Appl. Phys. Lett. 1999, 75, 1631-1633.

(4) Kölbel, M.; Huskens, J.; Brugger, J.; Reinhoudt, D. N. Proc. Micro Nano Eng. Conf. 2001, Grenoble, France, Sept 16-19, 2001; pp $350-351$. 
(5) Kölbel, M.; Tjerkstra, R. W.; Kim, G.; Brugger, J.; van Rijn, C. J. M.; Nijdam, W.; Huskens, J.; Reinhoudt, D. N. Adv. Funct. Mater., submitted for publication.

(6) Only insignificant height differences are observed by AFM and SEM when dots obtained with three uncoated stencils are compared.

(7) The stencil apertures and dots do not have a perfect circular contour but rather an elliptical contour. The diameters used in the calculations were obtained by averaging over the long and the short axes of the elliptical apertures and dots.

(8) AFM height measurements are more reliable since it is difficult to adjust the sample in a way that allows for imaging of the features by SEM under an angle of exactly $90^{\circ}$.
(9) $\mathrm{C} 12-\mathrm{Si}(\mathrm{OEt})_{3}$ and $\mathrm{C} 12-\mathrm{SiCl}_{3}$ give $\mathrm{SAMs}$ with nearly identical properties. The results obtained with $\mathrm{C} 12-\mathrm{SiCl}_{3}$ on stencils are, however, less reproducible. We assume this to be due to the higher reactivity and moisture sensitivity of the compound compared with the properties of $\mathrm{C} 12-\mathrm{Si}(\mathrm{OEt})_{3}$ and recommend that alkoxysilanes always be used.

(10) Because of diffusion and the scattering of gold across the surface, a porous gold film with a thickness of several nanometers is formed between the dots when $1-\mu \mathrm{m} \mathrm{Au}$ is evaporated.

NL025784O 